\title{
Ag Nanorod Arrays for SERS: Aspects of Spectral Reproducibility, Surface Contamination, and Spectral Sensitivity
}

\author{
Martin Šubr, ${ }^{1}$ Martin Petr, ${ }^{2}$ Vlastimil Peksa, ${ }^{1}$ Ondřej Kylián, ${ }^{2}$ \\ Jan Hanuš, ${ }^{2}$ and Marek Procházka ${ }^{1}$ \\ ${ }^{1}$ Institute of Physics, Faculty of Mathematics and Physics, Charles University in Prague, Ke Karlovu 5, 12116 Prague, Czech Republic \\ ${ }^{2}$ Department of Macromolecular Physics, Faculty of Mathematics and Physics, Charles University in Prague, \\ V Holešovičkách 2, 18000 Prague, Czech Republic \\ Correspondence should be addressed to Marek Procházka; prochaz@karlov.mff.cuni.cz
}

Received 25 November 2014; Accepted 17 February 2015

Academic Editor: Weihai Ni

Copyright (c) 2015 Martin Šubr et al. This is an open access article distributed under the Creative Commons Attribution License, which permits unrestricted use, distribution, and reproduction in any medium, provided the original work is properly cited.

\begin{abstract}
Ag nanorod arrays prepared by oblique angle vapor deposition (OAD) represent regular, large area substrates for surface-enhanced Raman scattering (SERS) spectroscopy. We studied uniformity and spectral reproducibility of silver OAD-fabricated substrates (AgOADs) by spectral mapping of methylene blue. The results demonstrate good reproducibility apart from occasional "hot-spot" sites where the intensity is higher. The number of "hot-spots" represents $2 \%-6 \%$ of SERS-active sites of mapping substrate area. We were able to obtain good SERS spectra of testing amino acid tryptophan at $1 \times 10^{-5} \mathrm{M}$ concentration and three different free-base porphyrins down to $\sim 10^{-7} \mathrm{M}$ concentration. We found out that keeping the AgOADs in a vacuum chamber overnight prevents the surface from binding any contaminants from the ambient atmosphere, without significant reduction in the SERS enhancement. Such substrates provide stable SERS enhancement even when stored for 1 year after preparation.
\end{abstract}

\section{Introduction}

Surface-enhanced Raman scattering (SERS) is a spectroscopic technique capable of detecting specific molecules at low concentrations $[1,2]$. It yields to enormous enhancement (above $10^{5}$ ) of Raman scattering of molecules placed in the vicinity of certain metal nanostructures. It is generally recognized that the dominant contribution to the SERS enhancement is due to the electromagnetic effect. This phenomenon is based on the resonance excitation of localized conductionelectron oscillations at the metal surface, which are termed surface plasmons [3]. The conditions for localized surface plasmon resonance (LSPR) are given by, namely, the size, shape, and degree of the aggregation of metal nanostructures $[1,3]$. In bioanalytical, medical, and biosensing applications of SERS (e.g., [2, 4-6]), the most commonly used metals are silver and gold because of their highly effective LSPR in the visible spectral region. The advantage of silver is the strongest enhancement allowing very low concentrations of analytes to be detected. Gold is due to its chemical inactivity more suitable for incorporation inside biological systems and/or designing of bioassay and biosensor platforms.

Roughened metal electrodes, metal island films, and metal colloids have been used in SERS spectroscopy since its discovery in the 70s. In attempts to fabricate more uniform and spectrally reproducible substrates, close attention has been paid to more sophisticated control of substrate morphology in the last $10-15$ years. The wide variety of employed preparation methods, including nanoparticle immobilization, nanoimprinting, templating, and nanosphere or nanocolloidal lithography, is reviewed, for example, in [7-9]. Chaney and coworkers developed a nanofabrication technique based on oblique angle vapor deposition (OAD) to obtain a versatile, simple, and inexpensive way of producing regular Ag nanorod arrays for SERS applications [10]. OAD utilizes an effect of self-shadowing 


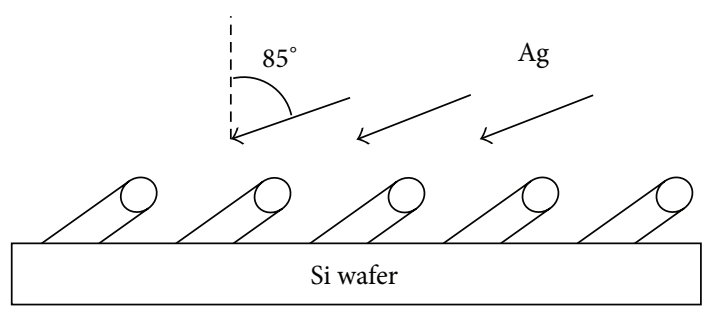

(a)

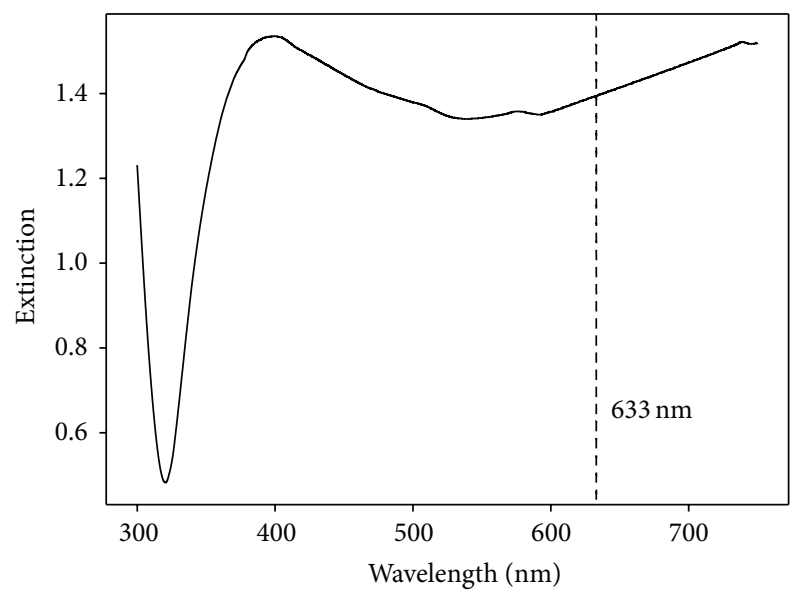

(b)

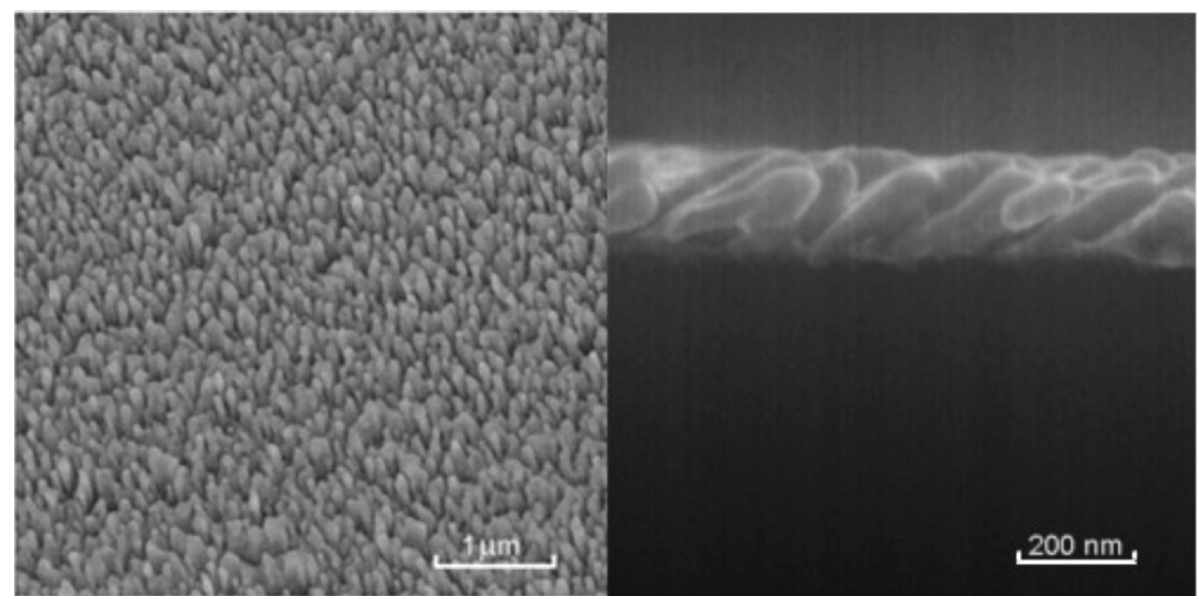

(c)

FIGURE 1: (a) Scheme of the OAD procedure, (b) extinction spectrum of prepared structures with excitation wavelength used for SERS measurements indicated by a dashed line, and (c) SEM images of a typical Ag nanorod array (top and side view).

which occurs when a collimated flux of vaporized atoms arrives at a substrate at an oblique angle with respect to the substrate normal. In this case, the growing nuclei shadow the area opposite to the incoming flux, and therefore the film grows through the formation of columnar structures separated from each other by voids and inclined towards the source of the flux (Figure 1(a)). The main advantage of OAD preparation is its simplicity. It is a one-step, vacuum-based process and, since no chemical substances are required, is also environmentally friendly. It was proved that OAD offers uniform, reproducible, large area SERS-active substrates with high SERS enhancement [11]. Ag nanorod arrays fabricated by OAD have been investigated as SERS-active substrates for the detection of pathogens, including viruses and bacteria, as well as assessing the potential of nucleotide-modified $\mathrm{Ag}$ nanorod arrays in a variety of biorecognition and biosensing applications [11].

In spite of recent advances in substrate fabrication suitable for SERS applications, employed metal nanostructures still pose a serious drawback for routine SERS quantitative analysis. In the case of Ag substrates, surface contamination irrespective of the nature of the fabrication method is often observed. It comes either from the preparation procedure or from the ambient atmosphere during storage and involves mainly organic (carbonaceous) species that are adsorbed on $\mathrm{Ag}$ surface. In the case of studied molecules with weak affinity to Ag surface, it can make their adsorption on the contaminated surface difficult or even impossible. Moreover, the surface contaminants (or impurities) manifest themselves, even in very small concentrations, by their anomalous SERS bands that overlap the SERS signal of a studied molecule. Such problems were previously reported in the case of both colloidal NPs $[12,13]$ and solid substrates $[9,14,15]$. Thus, special care must be exercised when analyzing any spectral pattern in an effort to reliably distinguish the bands coming from the analyte from those of the contaminants or, better still, to develop a method for preventing the contamination. There have been numerous ways to clean SERS-active surfaces from contaminants under ambient conditions, including electrochemical [9], plasma [14, 15], or ozone cleaning [16], but none have been uniformly successful [11]. Unfortunately, a side result of some electrochemical desorption 
techniques can be the introduction of surface defects where the carbon contamination was present or strong oxidation due to the high chemical reactivity of $\mathrm{Ag}[16,17]$. In the case of Ag nanorod arrays prepared by the OAD technique, it was demonstrated that under controlled plasma conditions and exposure times ( $<4 \mathrm{~min}$.) the Ar plasma cleaning procedure essentially eliminated any detectable background organic and carbonaceous contamination from the surface without substantially changing their morphology [14]. Moreover, it was demonstrated that the process of ion etching was able to recover SERS effectiveness of such substrates even after 1month storage [15].

In this paper, we focused on testing of silver OADfabricated nanorod array substrates (AgOADs) for SERS spectroscopy. We studied uniformity and spectral reproducibility of AgOADs by spectral mapping of methylene blue. We were able to obtain SERS spectra of biomolecules tryptophan down to $1 \times 10^{-5} \mathrm{M}$ concentration and porphyrins down to $\sim 10^{-7} \mathrm{M}$. We found out that keeping the substrates in a vacuum chamber overnight prevents it from binding any further contaminants from the ambient air, moreover, without significant reduction in the SERS enhancement.

\section{Materials and Methods}

2.1. Materials. Methylene blue (MB), L-tryptophan, 5, $10,15,20$-tetrakis (1-methyl-4-pyridyl)-21H,23H-porphine $\left(\mathrm{H}_{2} \mathrm{TMPyP}\right), \quad 5,10,15,20$-tetrakis(4-trimethylammoniophenyl)-21H,23H-porphine ( $\mathrm{H}_{2}$ TMAP), and 5,10,15,20-tetrakis(4-sulfonatophenyl)-21H,23H-porphine ( $\left.\mathrm{H}_{2} \mathrm{TSPP}\right)$ were purchased from Sigma-Aldrich.

2.2. Preparation and Characterization of $A g O A D s$. For preparation of the AgOADs, a magnetron sputtering of silver target was used (Figure 1(a)). Depositions were performed in a cylindrical stainless steel vacuum chamber (401) pumped by scroll and turbomolecular pumps to base pressure lower than $10^{-3} \mathrm{~Pa}$. Films were deposited onto $\mathrm{Si}$ wafer support introduced into the deposition chamber by a load-lock system. Ar (purity 99.99\%) was used as a working gas. The pressure during deposition was $0.18 \mathrm{~Pa}$, magnetron current was $300 \mathrm{~mA}$, distance between magnetron and substrate was $10 \mathrm{~cm}$, deposition angle was $85^{\circ}$, and deposition time was 15 minutes. Under these conditions the mean diameter of individual Ag nanorods was $60 \mathrm{~nm}$, the mean distance between their centers was $150 \mathrm{~nm}$, and the height of the nanorod array was around $200 \mathrm{~nm}$ as can be seen in Figure 1(c) where top and side views of Ag nanorods acquired by scanning electron microscopy (SEM, TESCAN Mira 3, $15 \mathrm{kV}$ accelerating voltage) are presented. For the sake of characterization of the surface plasmon properties of the AgOADs, silver deposition was performed on glass and extinction spectra of structures fabricated in this way were obtained (UVVis spectrophotometer Hitachi U-3300). From Figure 1(b) it follows that the AgOADs exhibit plasmon resonance over a very broad range of wavelengths.

2.3. SERS Measurements. For spectral reproducibility measurements, the AgOADs (deposited on Si wafer) were cut into $\sim 0.5 \mathrm{~cm} \times 0.5 \mathrm{~cm}$ pieces, immersed in $1 \times 10^{-6} \mathrm{M}$ stock solution of $\mathrm{MB}$ for 1 hour, and then removed and dried with an air stream. For SERS measurements of tryptophan and porphyrins, a small drop of sample was deposited on the Ag surface and left to dry. SERS spectra were collected at room temperature using an integrated confocal Raman microscopic system LabRam HR800 (Horiba Jobin-Yvon), equipped with a diffraction grating with 300 grooves $/ \mathrm{mm}$ and a liquid nitrogen cooled CCD detector. We tried $514.5 \mathrm{~nm}, 632.8 \mathrm{~nm}$, and $785 \mathrm{~nm}$ excitation wavelengths, but the $632.8 \mathrm{~nm}$ one provided the best SERS signal. Thus, HeNe laser, operating at the wavelength $632.8 \mathrm{~nm}$, was used as an excitation source for all SERS measurements. Laser power at the sample was $0.07 \mathrm{~mW}, 0.02 \mathrm{~mW}$, and $0.002 \mathrm{~mW}$ for $\mathrm{MB}$, tryptophan, and porphyrins, respectively. The laser beam was focused to a spot of about $1 \mu \mathrm{m}$ diameter using 100x objective; $\mathrm{NA}=0.9$. Scattered radiation was collected in a backscattering geometry and filtered by an edge filter for Rayleigh rejection before focusing it onto the $100 \mu \mathrm{m}$ entrance slit of the spectrometer. Acquisition times were $1 \mathrm{~s}, 10 \mathrm{~s}$, and $60 \mathrm{~s}$ for $\mathrm{MB}$, tryptophan, and porphyrins, respectively.

\section{Results and Discussion}

3.1. Aspects of Spectral Reproducibility. Uniformity and spectral reproducibility of the prepared AgOADs were evaluated by spectral mapping of MB, which is a well-established SERS probe molecule and a very efficient Raman scatterer (for its chemical structure see Figure 2). We tested different batches of AgOADs by mapping of random places at the samples covering $10 \times 10$ spectral points. Spectral maps were processed by in-house developed software based on factor analysis (FA), using a singular value decomposition (SVD) algorithm [18]. This procedure provides a set of singular values $W_{j}$, orthonormal subspectra $S_{j}(t)$, and a unitary matrix of corresponding $V_{i j}$ coefficients. A particular experimental spectrum $Y_{i}(t)$ within the treated series $(i=1, \ldots, N)$ can be expressed as

$$
Y_{i}(t)=\sum_{j=1}^{m} W_{j} V_{i j} S_{j}(t) .
$$

The factor dimension $m$ can be determined as the number of independent components (subspectra) resolvable in the analyzed spectral set and its value can be derived from the plot of the residual error values against the subspectra numbers. The $W_{j}$ is a vector representing the relative statistical weight of each subspectrum and the $V_{i j}$ is a unitary matrix representing relative contents of a subspectrum $S_{j}(t)$ in a particular spectrum $Y_{i}(t)$. In our case, spectra obtained in different mapping points may vary in intensities but not in the shape of the basic spectral profile (in an ideal case, all spectra in different mapping points are just multiples of one basic spectral profile). That is why we may assume with sufficient preciseness that $m=1$ with only the first subspectrum $S_{1}$ being relevant for our further calculations, whereas other subspectra represent only noise or at most slight changes in the background, traces of parasitic signal, and other artifacts. Thus, we used the coefficients $V_{i 1}$ to construct spectral maps of $\mathrm{MB}$. 

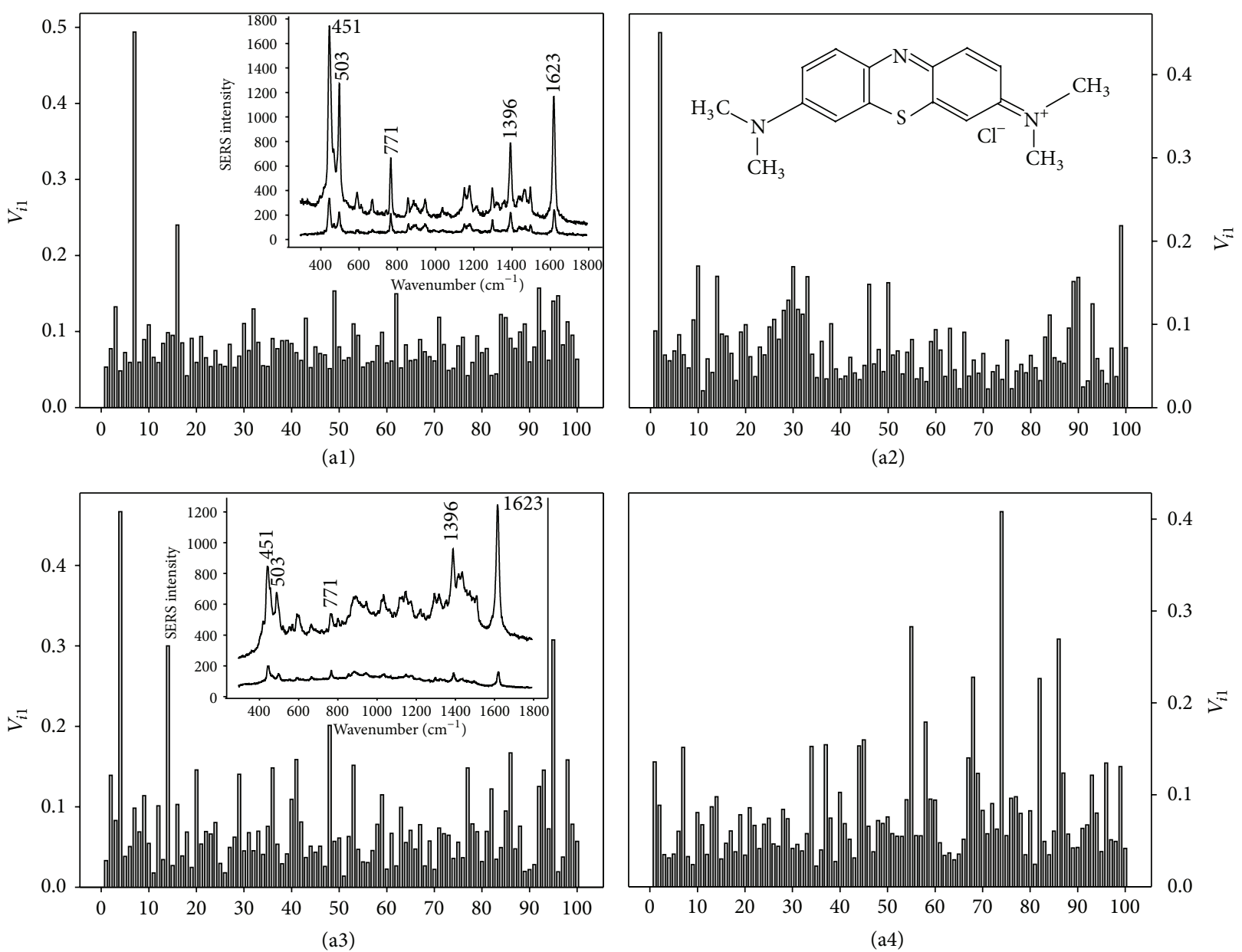

(a)

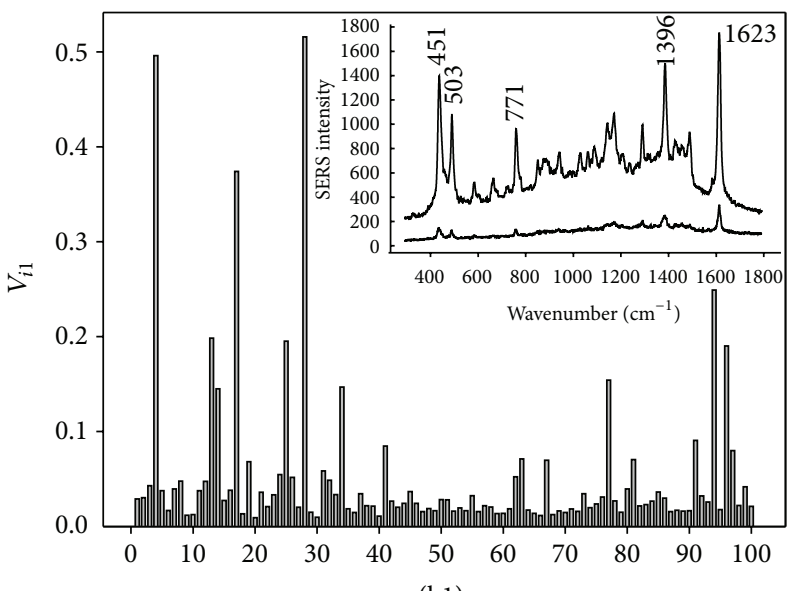

(b1)

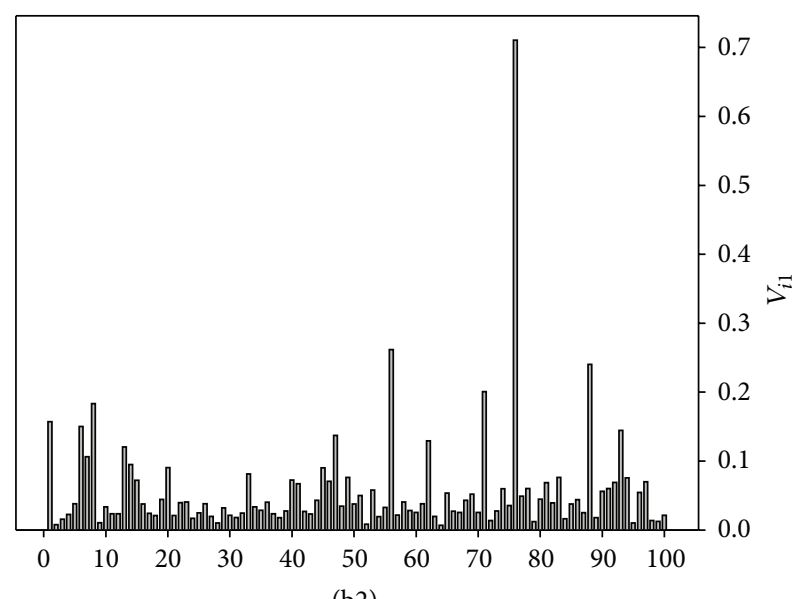

(b)

FIGURE 2: Reproducibility of spectral maps of MB on two different batches of AgOADs ((a) and (b)) in terms of coefficients $V_{i 1}$. Steps between mapping points were $5 \mu \mathrm{m}$. Spectral maps on the batch (a) were taken two days after their fabrication ((a1), (a2)) and after 1-year storage ((a3), (a4)). Examples of MB spectra with an average enhancement and spectra obtained from "hot-spot" sites are depicted as insets of graphs (al), (a3), and (b1). Chemical structure of MB is depicted as inset of graph (a2). 


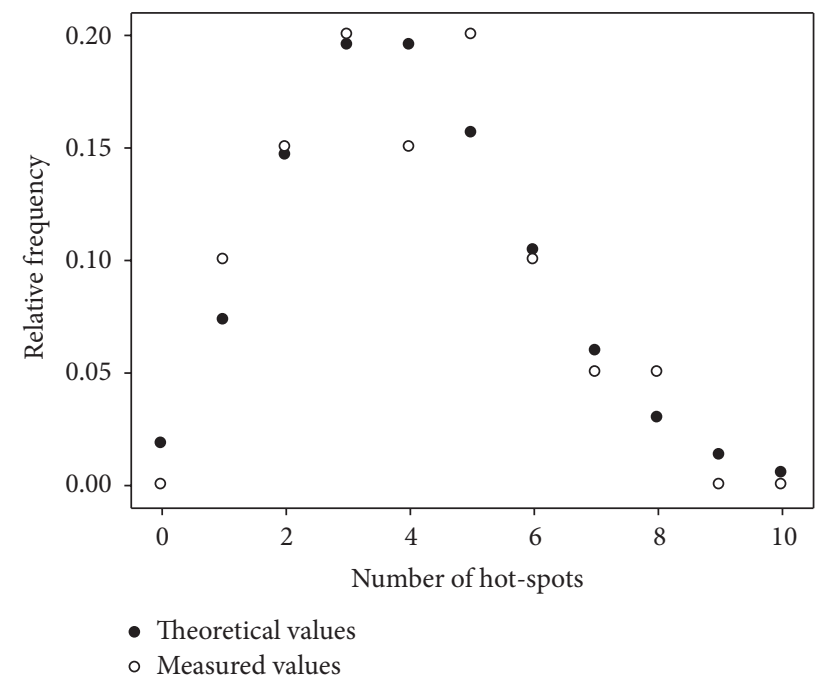

FIgURE 3: Comparison of the relative frequency of number of "hotspots" in a given map of $10 \times 10$ spectral points (out of 20 maps in total) with the values predicted by the Poisson distribution with fixed mean value of 4 .

Comparison of spectral reproducibility and SERS effectiveness of two different batches of AgOADs ((a), (b)) and after different storage times ( 2 days and 1 year) on a given batch are depicted in Figure 2. It is clear that both batches as well as 2-day and 1-year aged substrates provide reproducible SERS enhancement, signal variation up to $20 \%$ apart from occasional points where the intensity is higher. Higher SERS intensity obviously comes from excitation of "hot-spot" sites, which are sparsely distributed over the surface. Thus, reproducibility of a given spectral map is influenced mainly by the presence of occasional "hot-spots"; however, the actual number of "hot-spots" in a given set of $10 \times 10$ spectral points fluctuates (see maps (a) and (b) in Figure 2). We determined the relative frequency of number of "hot-spots" in a mapping area ( $10 \times 10$ spectral points) using 20 spectral maps of $\mathrm{MB}$, exhibiting the expected Poisson distribution (Figure 3). We assumed that "hot-spot" is a point where the intensity was at least 3 times the average intensity of a given spectral map. The mean number of "hot-spots" in a given set is $\sim 4$ and the mean deviation is $\sim 2$. Thus, we conclude that the number of "hotspots" represents $2 \%-6 \%$ of SERS-active sites of the mapping substrate area.

\subsection{Aspects of Surface Contamination and SERS Sensitivity.} In order to evaluate the SERS sensitivity of AgOADs, we tested amino acid tryptophan and porphyrins. They represent biomolecules widely studied by means of SERS. We measured their SERS spectra by depositing a droplet of a stock solution on the AgOADs and left it to dry. This process resulted in rather irregular distribution of molecules on the metal surface, leading to the loss of reproducibility of obtained spectra across different mapping points (in comparison to Figure 2). On the other hand, increased concentration of the analyte in certain regions of the substrate can increase the SERS sensitivity.

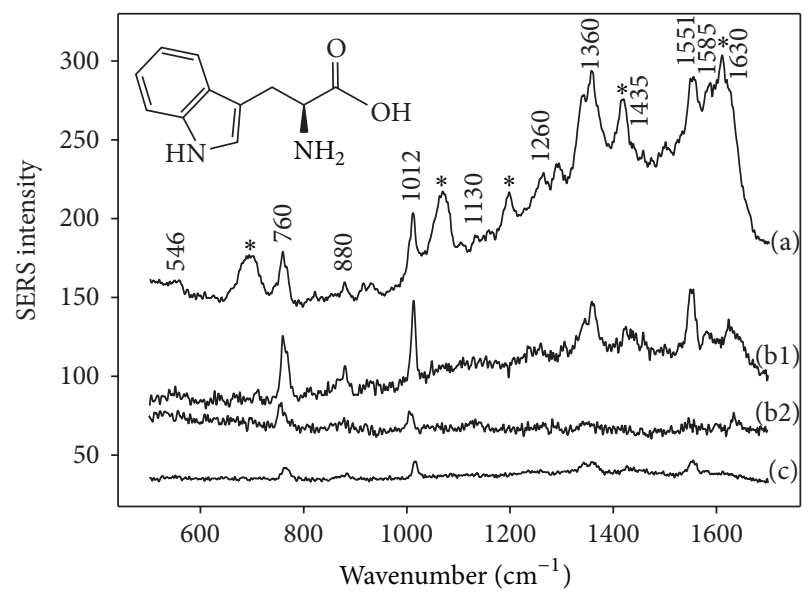

FIGURE 4: SERS spectra of tryptophan on two different AgOADs and demonstration of the effect of cleaning: (a) $1 \times 10^{-4} \mathrm{M}$ tryptophan on contaminated substrate, (b) tryptophan on clean substrate kept in vacuum overnight, $1 \times 10^{-4} \mathrm{M}(1)$ and $1 \times 10^{-5} \mathrm{M}$ concentration (2), and (c) $1 \times 10^{-4} \mathrm{M}$ tryptophan on contaminated substrate after 5 min. ultrasonic cleaning. Positions of main anomalous bands are marked with asterisks. Inset: chemical structure of tryptophan.

As already mentioned, one of the major limitations of exploiting AgOADs for SERS applications is the contamination of their surface by organic contaminants. This problem may be overcome by specific cleaning of the substrate, for example, by Ar plasma [14, 15] or ozone [16] treatment. However, the success of these methods strongly depends on the time of the applied cleaning and may lead to a reduction in SERS effectiveness due to suffered oxidation damage or distortion of the surface nanostructures. In the case of $\mathrm{MB}$ measurements no anomalous SERS bands of impurities were observed probably because MB molecules have a high affinity to silver and are able to replace the contaminants from the surface. On the other hand, when tryptophan was measured, we found a strong interference of surface contaminant bands with the tryptophan spectrum. Thus, we tried to find a way of cleaning the Ag surface before deposition of tryptophan. Figure 4 demonstrates the SERS spectra of tryptophan on (a) substrates exposed to the ambient atmosphere immediately after their fabrication, exhibiting anomalous bands marked with asterisks, (b) substrates kept in vacuum overnight, and (c) substrates after a 5 min. ultrasonic treatment.

Although the ultrasonic treatment did in some cases lead to the disappearance of anomalous bands from the spectra, the SERS effectiveness was approximately $4 \mathrm{x}$ worse. Keeping the substrates several hours in vacuum immediately after the deposition managed to reduce the spectra of contaminants significantly without a significant decrease in the SERS enhancement. By extending the time for which the substrates were kept in a vacuum after the deposition, SERS intensity of anomalous bands was gradually decreasing with almost complete suppression of a parasitic signal after overnight storage in a vacuum. We suggest that keeping the substrates in a vacuum chamber for at least 16 hours prevents it from binding any further contaminants in the ambient air. Such 


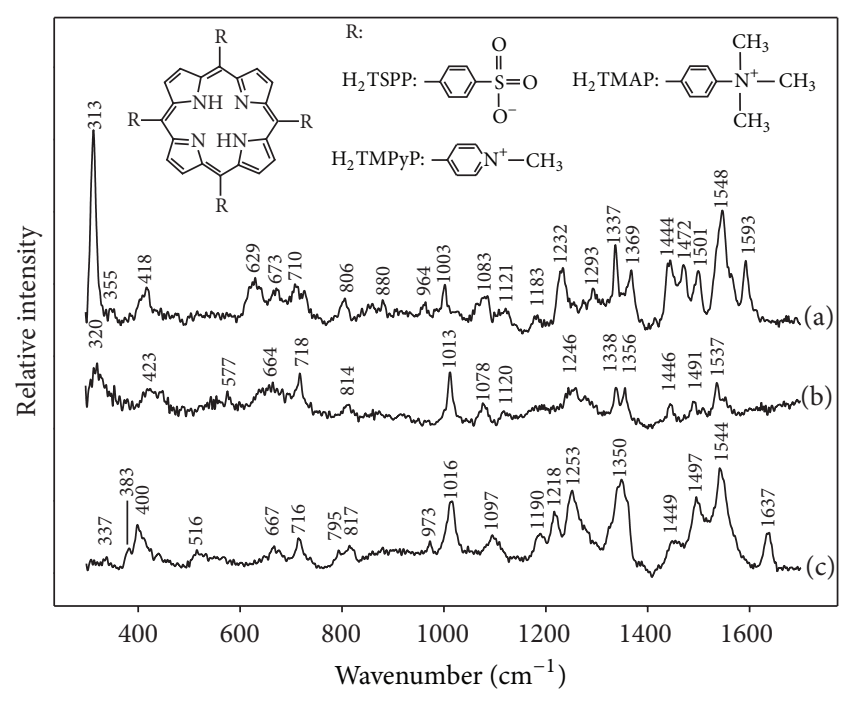

FIGURE 5: SERS spectra of three free-base porphyrins on AgOADs: (a) $\mathrm{H}_{2}$ TSPP, $3 \times 10^{-7} \mathrm{M}$, (b) $\mathrm{H}_{2}$ TMAP, $3 \times 10^{-7} \mathrm{M}$, and (c) $\mathrm{H}_{2}$ TMPyP, $5 \times 10^{-7} \mathrm{M}$ concentrations. Spectra are baseline corrected and shifted in vertical scale for the sake of clarity. Inset: chemical structure of porphyrins.

substrates provide stable enhancement even 3 months after their fabrication. We consider this method of keeping the substrates in a vacuum immediately after the deposition convenient due to the possibility of not using any other chemicals or further cleaning procedures whose influence on the surface morphology may be arguable.

The SERS spectra of tryptophan are dominated by two intense bands around 760 and $1012 \mathrm{~cm}^{-1}$ (Figure 4), which are assigned to the ring-breathing vibrations of the indole ring [19]. These two bands remain obvious down to $1 \times 10^{-5} \mathrm{M}$ concentration. Our tryptophan spectra are analogous to those reported previously using Ag nanoparticles [19, 20].

The final step of this study was testing of capability of fabricated clean AgOADs, that is, substrates that were kept 16 hours in vacuum after their deposition, to detect different porphyrins. The chemical structures of studied free-base porphyrins are depicted as inset of Figure 5. Two porphyrins $\left(\mathrm{H}_{2}\right.$ TMPyP and $\mathrm{H}_{2}$ TMAP) are positively charged and one is charged negatively $\left(\mathrm{H}_{2} \mathrm{TSPP}\right)$. SERS spectra of these three porphyrins of the order of $10^{-7} \mathrm{M}$ concentration were obtained from clean AgOADs (Figure 5). Our SERS spectra have a very good signal-to-noise ratio and positions of bands correspond very well with the spectra obtained previously using Ag nanoparticles [21, 22]. No anomalous bands of surface contaminants were observed.

\section{Conclusions}

We studied uniformity and spectral reproducibility of $\mathrm{Ag}$ nanorod arrays prepared by an oblique angle vapor deposition (OAD) technique. SERS spectra of methylene blue were used for spectral mapping through the AgOADs. The results demonstrated good reproducibility apart from occasional "hot-spot" sites where the intensity is higher. The number of "hot-spots" represents $2 \%-6 \%$ of SERS-active sites of mapping substrate area. Freshly prepared substrates exhibited strong anomalous bands from surface contaminants, often overlapping SERS spectra of the studied molecules. We found out that keeping the substrates in a vacuum chamber overnight after the preparation is a simple way to avoid this effect without a significant reduction in the SERS enhancement. Such substrates provide stable enhancement even after 1-year storage. We demonstrate that, by such substrate treatment, appropriate choice of laser power with respect to a given molecule, and reduced time of exposure to the laser beam, we were able to obtain SERS spectra of tryptophan at $1 \times 10^{-5} \mathrm{M}$ concentration and as low as $\sim 10^{-7} \mathrm{M}$ for porphyrins.

\section{Conflict of Interests}

The authors declare that there is no conflict of interests regarding the publication of this paper.

\section{Acknowledgments}

Funding of research Projects P205/13/20110S from the Czech Science Foundation and no. SVV 260092 from the Charles University in Prague is gratefully acknowledged.

\section{References}

[1] E. C. Le Ru and P. G. Etchegoin, Principles of Surface-Enhanced Raman Spectroscopy and Related Plasmonic Effects, Elsevier, Amsterdam, The Netherlands, 2009.

[2] S. Schlücker, "Surface-enhanced Raman spectroscopy: concepts and chemical applications," Angewandte Chemie International Edition, vol. 53, no. 19, pp. 4756-4795, 2014.

[3] M. Moskovits, "Surface-enhanced spectroscopy," Reviews of Modern Physics, vol. 57, no. 3, pp. 783-826, 1985.

[4] K. C. Bantz, A. F. Meyer, N. J. Wittenberg et al., "Recent progress in SERS biosensing," Physical Chemistry Chemical Physics, vol. 13, no. 24, pp. 11551-11567, 2011.

[5] D. Cialla, A. März, R. Böhme et al., "Surface-enhanced Raman spectroscopy (SERS): progress and trends," Analytical and Bioanalytical Chemistry, vol. 403, no. 1, pp. 27-54, 2012.

[6] M. Procházka and J. Štěpánek, "Surface-enhanced Raman scattering (SERS) and its application to biomolecular and cellular investigation," in Applications of Raman Spectroscopy to Biology_From Basic Studies to Disease Diagnosis, M. Ghomi, Ed., IOS Press, Amsterdam, The Netherlands, 2012.

[7] M. J. Banholzer, J. E. Millstone, L. D. Qin, and C. A. Mirkin, "Rationally designed nanostructures for surface-enhanced Raman spectroscopy," Chemical Society Reviews, vol. 37, no. 5, pp. 885-897, 2008.

[8] M. Fan, G. F. S. Andrade, and A. G. Brolo, "A review on the fabrication of substrates for surface enhanced Raman spectroscopy and their applications in analytical chemistry," Analytica Chimica Acta, vol. 693, no. 1-2, pp. 7-25, 2011.

[9] X.-M. Lin, Y. Cui, Y.-H. Xu, B. Ren, and Z.-Q. Tian, "Surfaceenhanced Raman spectroscopy: substrate-related issues," Analytical and Bioanalytical Chemistry, vol. 394, no. 7, pp. 1729-1745, 2009. 
[10] S. B. Chaney, S. Shanmukh, R. A. Dluhy, and Y.-P. Zhao, "Aligned silver nanorod arrays produce high sensitivity surfaceenhanced Raman spectroscopy substrates," Applied Physics Letters, vol. 87, no. 3, Article ID 031908, 2005.

[11] P. Negri and R. A. Dluhy, "Ag nanorod based surface-enhanced Raman spectroscopy applied to bioanalytical sensing," Journal of Biophotonics, vol. 6, no. 1, pp. 20-35, 2013.

[12] S. Sánchez-Cortés and J. V. García-Ramos, "Anomalous Raman bands appearing in surface-enhanced Raman spectra," Journal of Raman Spectroscopy, vol. 29, no. 5, pp. 365-371, 1998.

[13] N. R. Yaffe and E. W. Blanch, "Effects and anomalies that can occur in SERS spectra of biological molecules when using a wide range of aggregating agents for hydroxylamine-reduced and citrate-reduced silver colloids," Vibrational Spectroscopy, vol. 48, no. 2, pp. 196-201, 2008.

[14] P. Negri, N. E. Marotta, L. A. Bottomley, and R. A. Dluhy, "Removal of surface contamination and self-assembled monolayers (SAMs) from silver (Ag) nanorod substrates by plasma cleaning with argon," Applied Spectroscopy, vol. 65, no. 1, pp. 6674, 2011.

[15] N. Nuntawong, P. Eiamchai, B. Wong-Ek et al., "Shelf time effect on SERS effectiveness of silver nanorod prepared by OAD technique," Vacuum, vol. 88, no. 1, pp. 23-27, 2013.

[16] K. L. Norrod and K. L. Rowlen, "Removal of carbonaceous contamination from SERS-active silver by self-assembly of decanethiol," Analytical Chemistry, vol. 70, no. 19, pp. 42184221, 1998.

[17] S. Z. Zou, Y. X. Chen, B. W. Mao, B. Ren, and Z. Q. Tian, "SERS studies on electrode/electrolyte interfacial water I. Ion effects in the negative potential region," Journal of Electroanalytical Chemistry, vol. 424, no. 1-2, pp. 19-24, 1997.

[18] J. Hanzlíková, M. Procházka, J. Štěpánek, J. Bok, V. Baumruk, and P. Anzenbacher Jr., "Metalation of 5,10,15,20-tetrakis(1methyl-4-pyridyl)porphyrin in silver colloids studied via time dependence of surface-enhanced resonance Raman spectra," Journal of Raman Spectroscopy, vol. 29, no. 7, pp. 575-584, 1998.

[19] C.-H. Chuang and Y.-T. Chen, "Raman scattering of Ltryptophan enhanced by surface plasmon of silver nanoparticles: vibrational assignment and structural determination," Journal of Raman Spectroscopy, vol. 40, no. 2, pp. 150-156, 2009.

[20] P. Šimáková, M. Procházka, and E. Kočišová, "SERS microspectroscopy of biomolecules on dried Ag colloidal drops," Spectroscopy, vol. 27, no. 5-6, pp. 449-453, 2012.

[21] M. Procházka, J. Hanzliková, J. Štěpánek, and V. Baumruk, "Metalation of positively charged water soluble mesoporphyrins studied via time-resolved SERRS spectroscopy," Journal of Molecular Structure, vol. 410-411, pp. 77-79, 1997.

[22] M. Itabashi, K. Kato, and K. Itoh, "Electrochemical processes of meso-tetrakis (4-sulfonatophenyl) porphine at a silver electrode studied by surface-enhanced resonance Raman spectroscopy," Chemical Physics Letters, vol. 97, no. 6, pp. 528-532, 1983. 

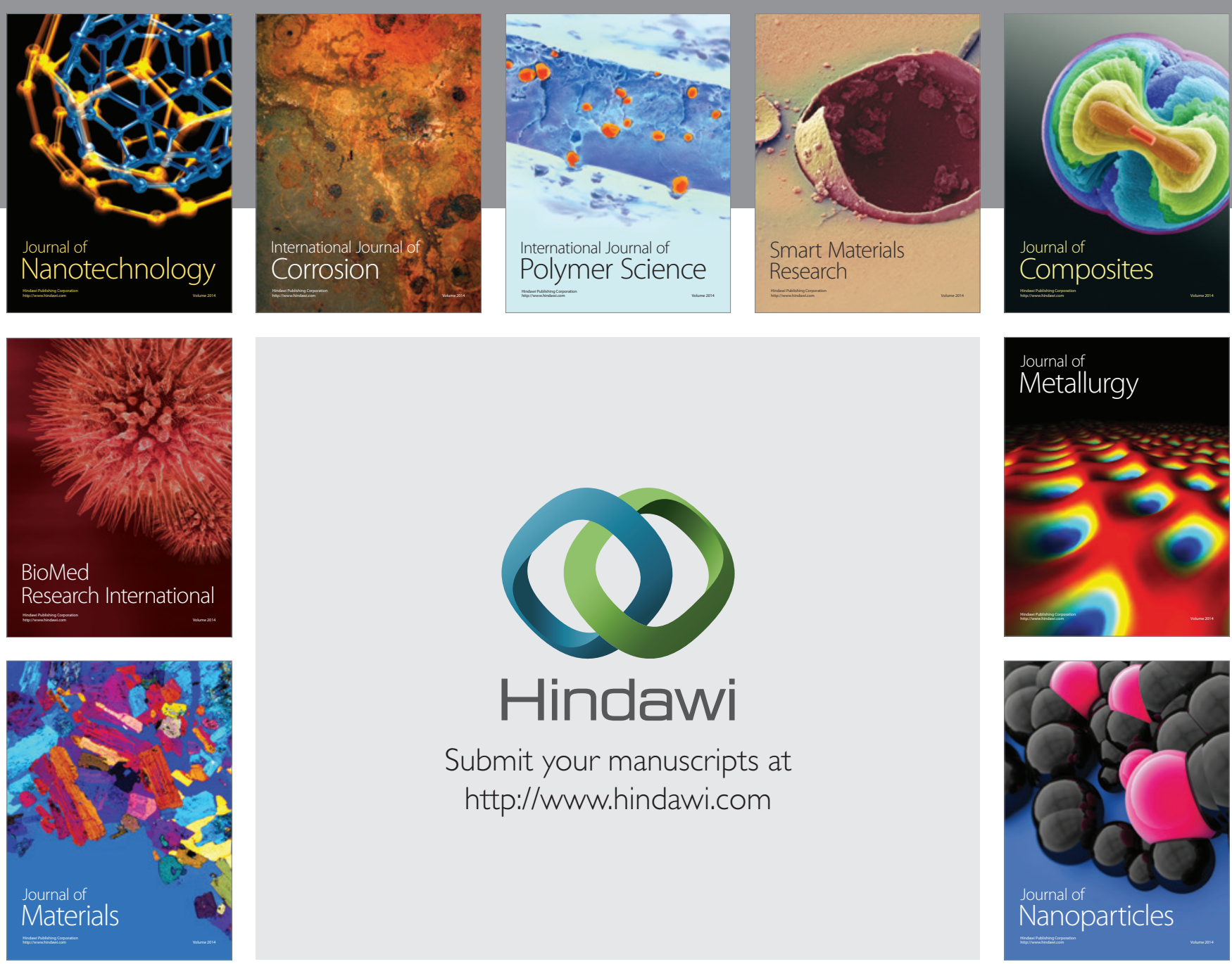

Submit your manuscripts at http://www.hindawi.com
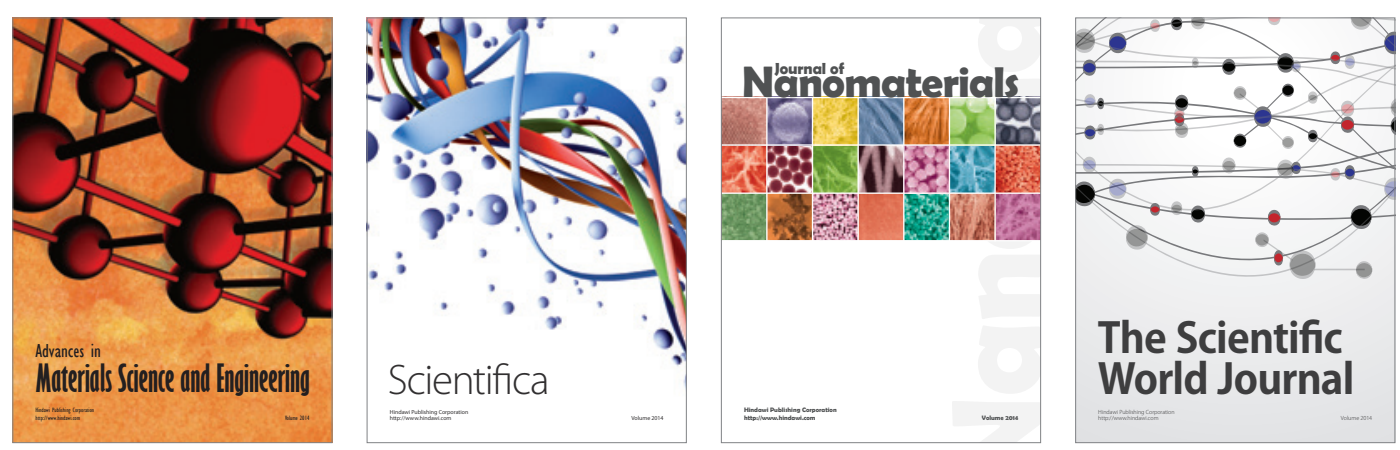

\section{The Scientific World Journal}
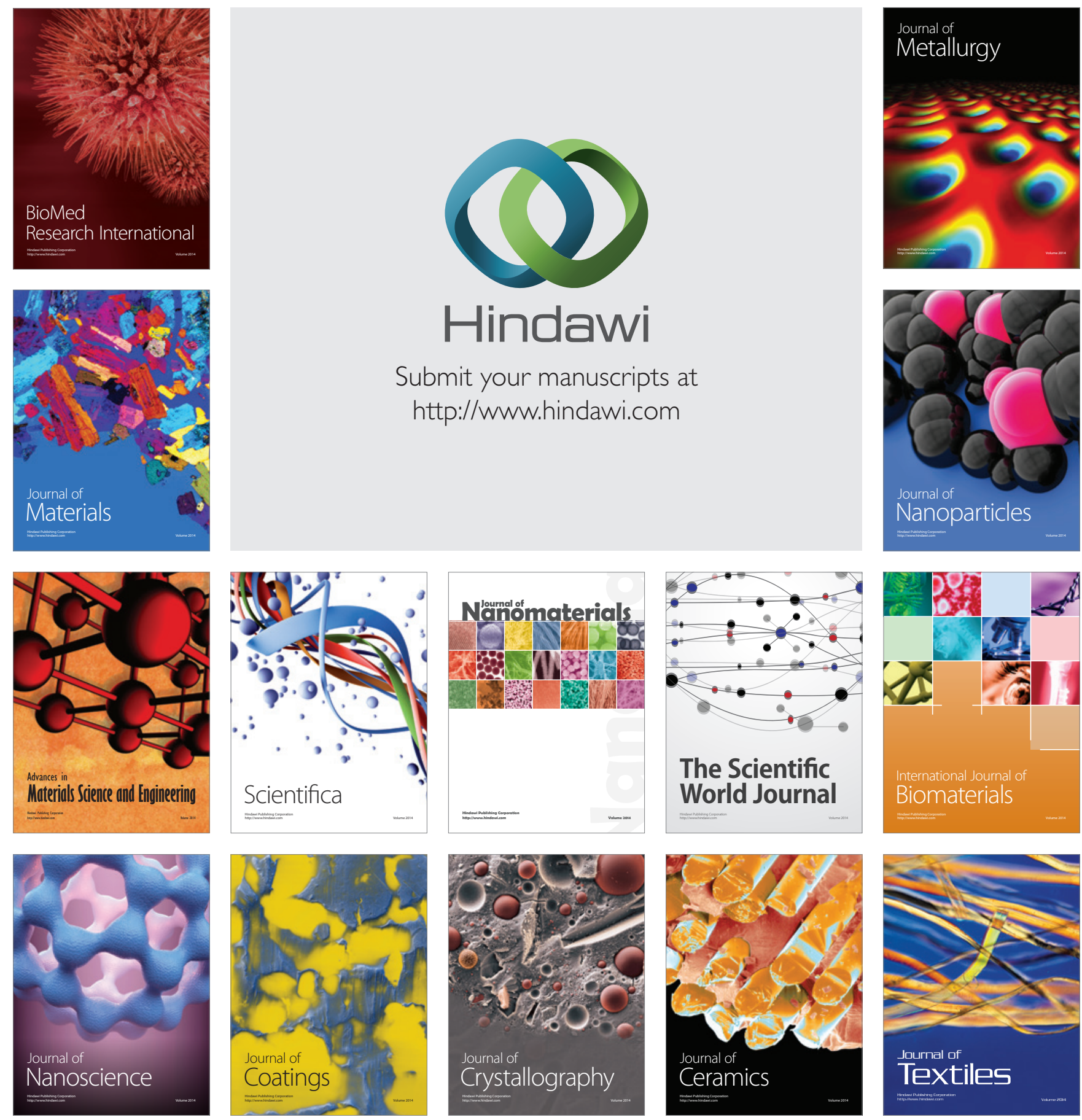